Trauma Berufskrankh 2016 · [Suppl 3]: 18:S254-S259

DOI 10.1007/s10039-015-0080-y

Online publiziert: 21. September 2015

๑) Springer-Verlag Berlin Heidelberg 2015

CrossMark

B. Schliemann - M. Herbort · S. Lenschow - M.J. Raschke · C. Kösters

Klinik für Unfall-, Hand- und Wiederherstellungschirurgie, Universitätsklinikum Münster,

Münster, Deutschland

\title{
Dynamische intraligamentäre Stabilisierung frischer Rupturen des vorderen Kreuzbandes (Ligamys)
}

\section{Umdenken in der Kreuzbandchirurgie?}

\section{Prinzip der dynamischen intraligamentären Stabilisierung}

Die Ruptur des vorderen Kreuzbandes (VKB) stellt eine der häufigsten Verletzungen des jungen, sportlich aktiven $\mathrm{Pa}$ tienten dar. Die Ersatzplastik mittels körpereigener Sehne hat sich zum Standardverfahren in der Behandlung der VKBRuptur und -insuffizienz entwickelt [3, 4, 9]. Durch Optimierung der Operationstechnik mit anatomischer Bohrkanalplatzierung über das anteromediale Portal ließen sich die vorher bereits guten Ergebnisse weiter verbessern $[1,2]$. Nachteile der Ersatzplastik sind jedoch die Zusatzmorbidität durch die Sehnenentnahme und mögliche Tunnelfehlplatzierungen, die eine chronische Instabilität begünstigen können. Ebenso können die propriozeptiven Eigenschaften, die dem intakten VKB zugeschrieben werden, durch eine Ersatzplastik nicht wiederhergestellt werden.

Eine Alternative zur Ersatzplastik bietet die primäre Naht des VKB. Marshall $[13,14]$ beschrieb 1979 eine Technik, bei der er die femoralen Stümpfe des rupturierten VKB über transossäre Ausziehnähte unter Berücksichtigung der anteromedialen und posterolateralen Bündel femoral refixierte. Umliegendes Synovialgewebe nähte er auf das reinserierte Band, um eine ausreichende Blutversorgung $\mathrm{zu}$ gewährleisten. Die Ergebnisse waren allerdings mäßig. Paessler et al. [15] erkannten die Notwendigkeit, die primä- re Naht zusätzlich abzusichern, und augmentierten das VKB nach Marshall-Naht mit einem synthetischen Band, das sie mit 50 N vorspannten. Nach einem Follow-up von 38 Monaten fanden sie eine Seitendifferenz im Lachman-Test von 2,4 mm (präoperativ 8,6 mm). Dennoch konnten nur $51 \%$ der Patienten zu ihrem ursprünglichen sportlichen Niveau zurückkehren.

Das Prinzip der Augmentation wurde auch für die Versorgung des zentralen Pfeilers bei Kniegelenkluxation als sog. „ligament bracing“ vorgeschlagen $[6,7]$. Allen Augmentationstechniken ist jedoch gemein, dass es sich um rigide und mehr oder weniger statische Verfahren handelt. Lubowitz [12] konnte jedoch zeigen, dass sich die Länge eines anatomisch platzierten VKB-Transplantates beim Bewegen des Kniegelenkes von $0^{\circ}$ Extension bis $120^{\circ}$ Flexion um durchschnittlich 6,7 mm ändert. Diese Längenänderung wird durch rigide Augmentationen nicht berücksichtigt. Bei der dynamischen intraligamentären Stabilisierung (DIS) dagegen wird der Augmentationsfaden mit einer Feder verbunden, die eine Exkursion von $8 \mathrm{~mm}$ erlaubt (• Abb. 1). Somit kann die angelegte Vorspannung während des gesamten Bewegungszyklus gehalten werden, Zug- und Scherkräfte werden minimiert, und die adaptierten Kreuzbandstümpfe verbleiben in derselben Position zueinander. Das Knie verbleibt in einer hinteren Schublade, so- dass das genähte VKB entlastet wird und potenziell heilen kann.

\section{Indikationen}

Für die DIS sind akute vordere Kreuzbandrupturen geeignet, die nicht älter als 21 Tage sind. Eine frische Ruptur ist für die Heilung adaptierter Bandenden essenziell. Zwar existieren keine exakten Daten zum spätestmöglichen Operationszeitpunkt, ein Intervall von 3 Wochen zur Versorgung frischer Band- und Sehnenverletzungen mittels Naht wird jedoch auch für andere Lokalisationen, z. B. akute Luxationen des Schultereckgelenkes, beschrieben.

Als relative Kontraindikationen werden daher VKB-Rupturen angesehen,

\begin{tabular}{|c|c|c|}
\hline & \multicolumn{2}{|c|}{ Follow-up (Monate) } \\
\hline & 12 & 24 \\
\hline $\mathrm{N}$ & 176 & 62 \\
\hline \multirow[t]{2}{*}{ Lysholm (Punkte) } & 96,07 & 97 \\
\hline & $( \pm 6,46)$ & $( \pm 5)$ \\
\hline \multirow[t]{2}{*}{ Tegner } & 5,1 & 5,1 \\
\hline & $( \pm 1,4)$ & $( \pm 1,4)$ \\
\hline \multirow{2}{*}{$\begin{array}{l}\text { International Knee } \\
\text { Documentation Com- } \\
\text { mittee (IKDC) (\%) }\end{array}$} & 93,5 & 94,8 \\
\hline & $( \pm 8,0)$ & $( \pm 6,5)$ \\
\hline \multirow[t]{2}{*}{ Satisfaction (VAS) } & 8,9 & 8,9 \\
\hline & $(1,3)$ & $(1,3)$ \\
\hline \multirow[t]{2}{*}{$\Delta$ Lachman (mm) } & 1,5 & 2,3 \\
\hline & $( \pm 1,7)$ & $( \pm 1,7)$ \\
\hline
\end{tabular}




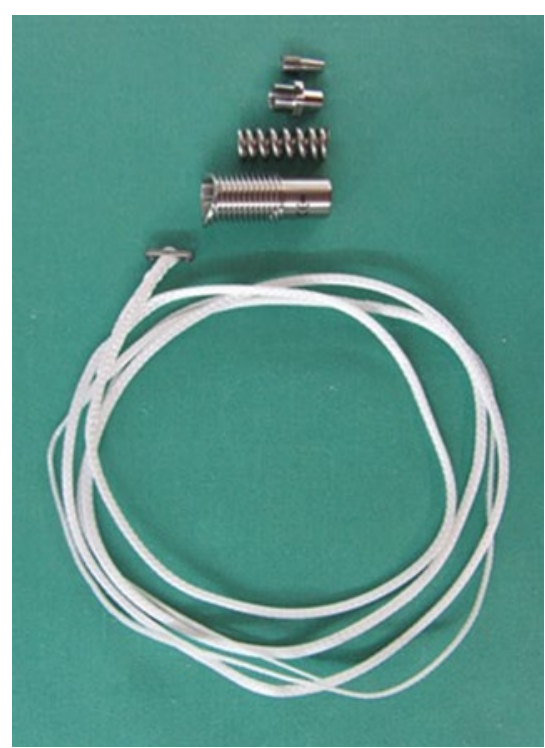

Abb. 1 ه Demontiertes Ligamys-Implantat: Monoblock, Feder, Klemmelement und Klemmschraube sowie Augmentationsfaden mit femoralem Button

bei denen das Trauma mehr als 3 Wochen zurückliegt. Akute oder chronische Infektionen (lokal oder systemisch), Arthrofibrose, schwere Achsabweichungen, schwere Muskel-, Nerven- oder Gefäßerkrankungen, Überempfindlichkeit gegenüber den verwendeten Werkstoffen (z. B. Kobalt, Chrom, Nickel usw.), ungenügende Knochensubstanz oder mangelhafte Knochenqualität, die eine stabile Verankerung des Implantates gefährden könnten, gelten als absolute Kontraindikationen.

Die Anwendung der DIS bei Kindern und Jugendlichen mit offenen Wachstumsfugen kann aufgrund fehlender klinischer Daten derzeit noch nicht ausreichend beurteilt werden und gilt somit aktuell als Kontraindikation.

\section{Operationstechnik}

Anästhesie, Lagerung und Portale entsprechen denen der arthroskopischen Kreuzbandersatzplastik. Für die Platzierung des femoralen Bohrkanals wird die Verwendung des anteromedialen Portals dringend empfohlen, um den femoralen Footprint sicher zu erreichen und eine Einheilung in anatomischer Position zu ermöglichen. Hierbei sollte das anteromediale Portal mit einer Größe von ca.
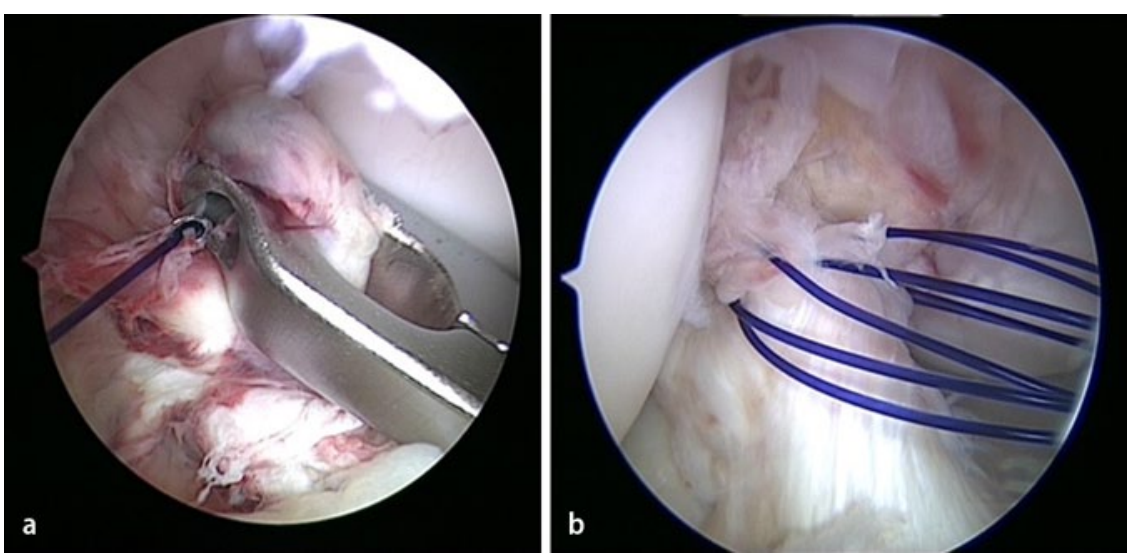

Abb. $2 \Delta$ Intraoperativer Befund. a Anschlingen des Stumpfes mittels Nahtzange. b Sämtliche Anteile des Stumpfes werden sukzessive armiert
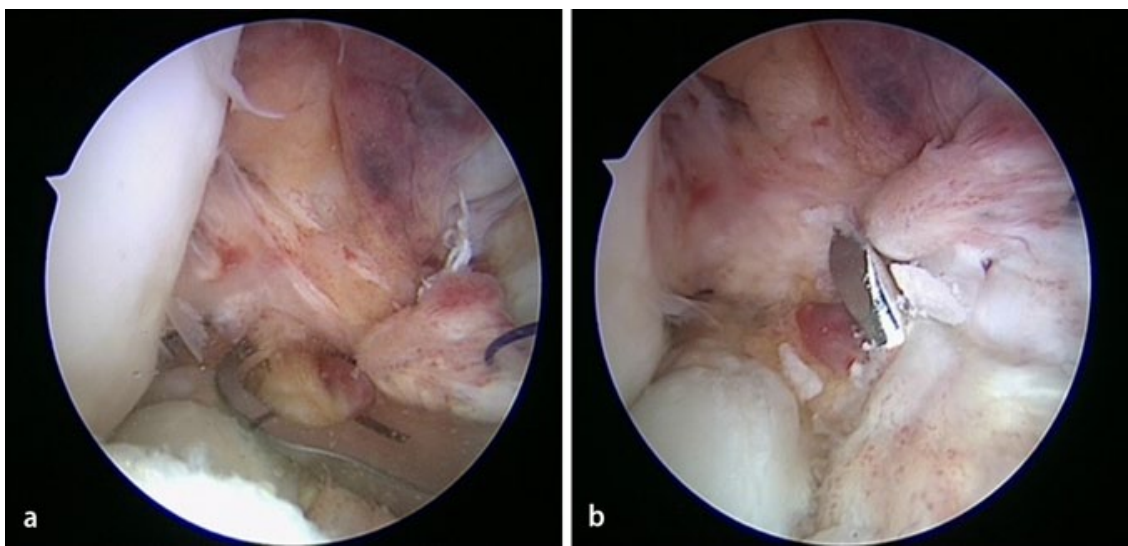

Abb. $3 \Delta$ a Einbringen des tibialen Zielgerätes unmittelbar hinter die tibiale Insertion. $\mathbf{b}$ Einbringen eines K-Drahtes

$1-1,5 \mathrm{~cm}$ angelegt werden, um ein problemloses Ein- und Ausführen der Nahtzange zu gewährleisten. Begleitverletzungen wie Meniskusrisse oder Knorpelschäden sollten versorgt werden, bevor mit der Kreuzbandnaht begonnen wird.

Nach Evaluation der Rupturform des VKB und ausführlicher Bilddokumentation wird der tibiale Stumpf des VKB mithilfe der Nahtzange mit 3 bis 4 (mindestens 2, maximal 5) Polydioxanon-(PDS-) Fäden der Stärke 2,0 armiert (• Abb. 2). Die Fäden werden aus dem anteromedialen Portal ausgeleitet.

Die am weitesten anterior gelegenen VKB-Anteile sollten zuerst angeschlungen werden, um eine bessere Kontrolle über den Stumpf zu ermöglichen. Durch leichten Zug am PDS-Faden kann der Stumpf nach vorne luxiert und die hinteren Anteile können somit besser erreicht werden. Schließlich sollten alle Anteile des Stumpfes erfasst sein.
Für die Platzierung des tibialen Monoblocks wird nun eine ca. $3 \mathrm{~cm}$ lange, schräg verlaufende Hautinzision etwas oberhalb des Pes anserinus angelegt und auf das Periost präpariert. Nun wird das tibiale Zielgerät über das anteromediale Portal eingeführt und ein kurzer, 2,3 mm starker Führungsdraht eingebracht. Um den tibialen Stumpf zu erhalten, wird der Führungsdraht knapp hinter dem tibialen Footprint platziert (• Abb. 3).

Nach arthroskopischer Kontrolle der korrekten intraartikulären Lage der Drahtspitze kann nun an der Skala der Bohrhülse die Distanz zwischen extraartikulärer Tibiakortikalis und intraartikulärem Footprint des VKB abgelesen werden. Eine Länge von $50 \mathrm{~mm}$ ist anzustreben. Im Falle einer Revision mit Wechsel auf eine Ersatzplastik verbleiben somit 20 mm unversehrter Knochen unterhalb der Gelenklinie, in dem das Transplantat einheilen kann. Anschließend wird die 
extraartikuläre Tibiakortikalis über den Führungsdraht mittels eines kanülierten 6-mm-Bohrers zunächst perforiert und die Bohrung anschließend mit dem tiefenbegrenzten 10-mm-Bohrer bis zu einer Tiefe von $30 \mathrm{~mm}$ aufgebohrt.

Gewebereste werden vom Tunneleingang entfernt, und der Monoblock mit innenliegender Feder wird über den Führungsdraht eingebracht (• Abb. 4a).

Im Anschluss wird der tibiale Führungsdraht entfernt und ein Shuttlefaden (z. B. 1er-Vicryl, mindestens $90 \mathrm{~cm}$ Länge) über den Monoblock unter arthroskopischer Sicht in das Gelenk eingebracht. Die Schlaufe wird ebenfalls durch das anteromediale Portal ausgeleitet. Zur Stammzellfreisetzung wird die laterale Notch mikrofrakturiert.

In $120^{\circ}$ Beugung wird nun ein Ösendraht über das femorale Zielgerät in den anatomischen femoralen Kreuzbandansatz platziert. Der Draht wird am lateralen distalen Oberschenkel ausgeleitet, die Haut inzidiert, die Muskulatur mittels eines Weichteildilatators aufgespreizt und eine Dilatatorhülse über den Draht geschoben, sodass diese bündig auf dem lateralen Femurkortex aufsitzt. Der Shuttlefaden und die PDS-Fäden, mit denen das VKB armiert ist, werden nun in die Öse des langen Führungsdrahts eingefädelt. Der lange Führungsdraht wird dann mit einem T-Griff-Bohrfutter vorsichtig mit allen Fäden durch das Femur nach proximal ausgeleitet.

Der mit einem Flipbutton versehene Augmentationsfaden wird nun mithilfe des Shuttlefadens durch das Gelenk gezogen und über den Monoblock ausgeleitet. Die PDS-Fäden werden dabei unter leichter Spannung gehalten ( $\bullet$ Abb. $\mathbf{4 b}$ ).

Durch das Straffen der Haltefäden und gleichzeitiges Anziehen des Augmentationsfadens nach distal werden die Kreuzbandstümpfe adaptiert.

Nach maximaler Vorspannung wird nun in voller Extension des Kniegelenkes ein Spanngerät auf den Monoblock aufgebracht und der Augmentationsfaden mit einer Vorspannung von ca. $80 \mathrm{~N}$ mit einem Klemmkonus im Federmechanismus verblockt (• Abb. 5). Eine zusätzliche Fixierung der Haltefäden ist nicht erforderlich, da es durch die Dicke des Augmentationsfadens sowie die Lage der Hal-

Trauma Berufskrankh 2016 · [Suppl 3]: 18:S254-S259 DOI 10.1007/s10039-015-0080-y

(c) Springer-Verlag Berlin Heidelberg 2015

\section{B. Schliemann · M. Herbort · S. Lenschow · M.J. Raschke · C. Kösters}

\section{Dynamische intraligamentäre Stabilisierung frischer Rupturen des vorderen Kreuzbandes (Ligamys). Umdenken in der Kreuzbandchirurgie?}

\section{Zusammenfassung}

Die Ersatzplastik des vorderen Kreuzbandes (VKB) mittels körpereigener Sehne hat sich als Standardverfahren in der Behandlung der VKB-Ruptur etabliert. Dennoch wurden immer wieder Versuche unternommen, das frisch rupturierte VKB zu nähen. Die Ergebnisse waren jedoch eher enttäuschend. Die dynamische intraligamentäre Stabilisierung (DIS) wurde entwickelt, um die biomechanische Stabilität nach primärer Naht des VKB zu gewährleisten und somit eine stabile Einheilung zu ermöglichen. Im Gegensatz zur einfachen Augmentation mittels nichtresorbierbarer Fäden wird bei der DIS ein Federmecha- nismus verwendet, um der Anisometrie des VKB gerecht zu werden und die Tibia über den gesamten Bewegungsumfang in der hinteren Schublade zu halten. Erste biomechanische und klinische Studien sind vielversprechend, eine erfolgreiche Wiederherstellung der Kniegelenkkinematik ist möglich. Weitere Studien sind allerdings notwendig, um die Wertigkeit dieses neuen Verfahrens für den klinischen Alltag zu belegen.

Schlüsselwörter

Kreuzbandinstabilität $\cdot$ Sehne $\cdot$ Stabilität .

Einheilung $\cdot$ Kniegelenkkinematik

\section{Dynamic intraligamentary stabilization of acute anterior cruciate ligament tears (Ligamys). Rethinking in cruciate ligament surgery?}

\section{Abstract}

Anterior cruciate ligament $(\mathrm{ACL})$ reconstruction with the use of autologous tendon grafts is the gold standard in the treatment of $A C L$ instability; however, many different techniques have been proposed to suture freshly ruptured ACLs. Unfortunately, the rate of recurrent instability is high. A new technique, dynamic intraligamentary stabilization (DIS), was developed to restore biomechanical stability after primary $\mathrm{ACL}$ repair and therefore to allow stable healing. In order to compensate for the anisometry of the $\mathrm{ACL}$, in contrast to simple augmentation a spring mechanism is used in DIS that helps to maintain the tibia in a posterior drawer position during all degrees of flexion. Initial results of biomechanical and clinical studies are promising, indicating that reconstruction of knee joint kinematics after $A C L$ repair with DIS is possible; however, further clinical studies are required to determine its usefulness in the clinical setting.

Keywords

Anterior cruciate ligament · Tendon .

Stability $\cdot$ Healing $\cdot$ Knee joint kinematics tefäden unter dem Flipbutton zu einer ausreichenden Verklemmung der Fäden kommt. Die PDS-Fäden werden unterhalb des Hautniveaus abgesetzt. Abschließend erfolgt die Kontrolle des Repositionsergebnisses in Flexion und Extension (- Abb. 6). Auf das Einlegen einer Drainage kann in der Regel verzichtet werden.

\section{Nachbehandlung}

Für die ersten 5 postoperativen Tage wird das operierte Bein in einer geraden Schiene immobilisiert. Um eine ausreichende Verklebung des refixierten Kreuzbandstumpfes über einen Blutklot zu erreichen, erfolgt in dieser Phase keine Beübung. In der Schiene ist eine sofortige schmerzadaptierte Vollbelastung möglich. Am ersten postoperativen Tag erfolgt eine Röntgenkontrolle in 2 Ebenen (- Abb. 7). Anschließend erfolgt die weitere Nachbehandlung orthesenfrei. Zunächst wird die freie Beweglichkeit erarbeitet. Vorsichtiges Krafttraining ist bereits ab der 4 . Woche möglich. Ab der 6. Woche wird mit dem Stabilitäts- und Koordinationstraining begonnen. Das Radfahren, auch auf der Straße, ist nun möglich. Laufen und sportartspezifisches Training kann ca. ab der 10. bis 12. Woche begonnen werden. Über die Rückkehr zum Sport muss nach entsprechenden Tests und abhängig von der Sportart individuell entschieden werden. 

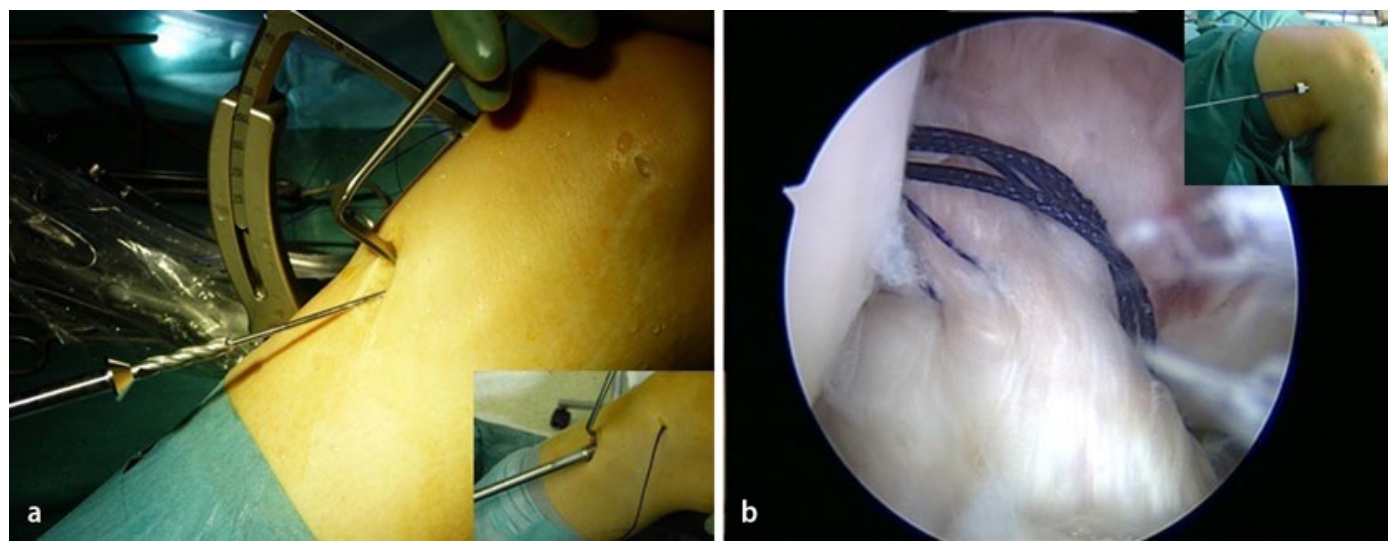

Abb. $4<$ a Über den Führungsdraht wird die proximale Tibia für die Platzierung des Monoblocks aufgebohrt. b Über einen Shuttlefaden werden die Polydioxanon-(PDS-)Fäden femoral ausgeleitet, der Augmentationsfaden gegenläufig durch das $\mathrm{Ge}$ lenk in den tibialen Monoblock eingezogen. (Aus [11])

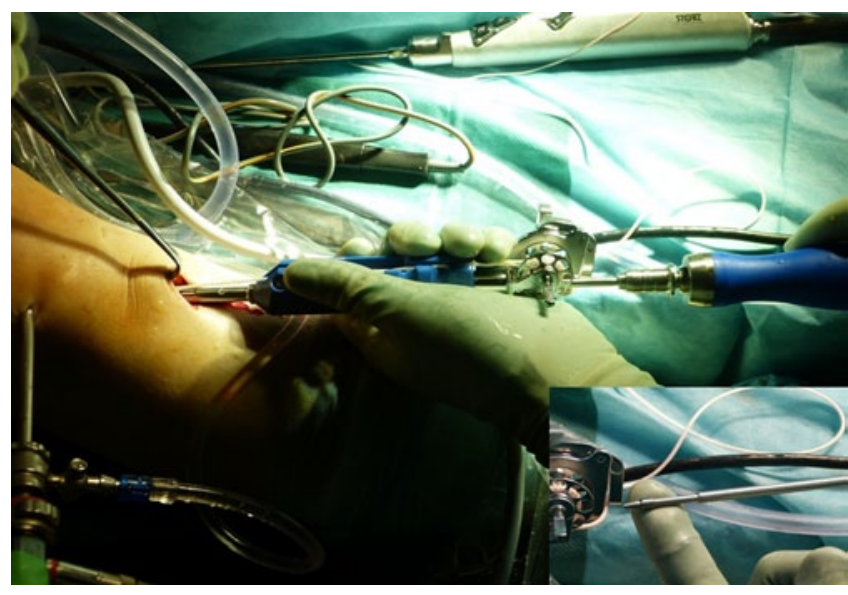

Abb. $5<$ Extensionsnah wird das Spanngerät aufgesetzt, eine Vorspannung von $80 \mathrm{~N}$ appliziert und der Faden im Klemmelement verblockt. (Aus [11])
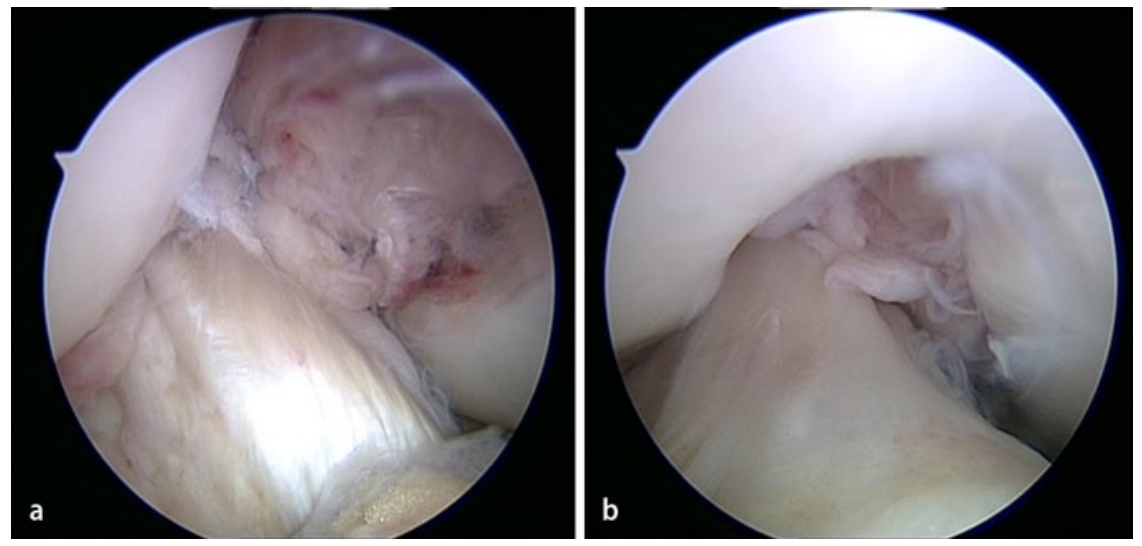

Abb. $6 \Delta$ Intraoperatives Ergebnis a in Flexion und $\mathbf{b}$ in Extension

Liegen Begleitverletzungen vor, die ebenfalls adressiert wurden, muss das Nachbehandlungsregime ggf. angepasst werden.

\section{Bisherige Ergebnisse und Ausblick}

In einer ersten Grundlagenarbeit am Schafmodell konnte bereits gezeigt werden, dass eine Heilung des rupturierten ten Lachman- und Pivot-shift-Test zeigten.

Nach einer klinischen Pilotstudie an 10 Patienten liegen mittlerweile auch mittelfristige Ergebnisse von über 250 Patienten vor [5]. Henle et al. [8] konnten für 278 Patienten mit einem Durchschnittsalter von 32,4 Jahren (18 bis 63) und einer mittleren Zeit zwischen Trauma und Operation von 18 Tagen sehr gute Ergebnisse in den klinischen Scores und eine hohe Patientenzufriedenheit nachweisen. Nach 24 Monaten betrug die Differenz im Lachman-Test im Vergleich zum gesunden Kniegelenk lediglich 2,3 mm (- Tab. 1). In der Zwischenzeit wurden 8 Patienten bei Reruptur und 3 Patienten bei Insuffizienz des VKB operativ mittels einzeitiger Sehnenplastik revidiert.

In unserer Klinik wurden seit Januar 2013 ca. 180 Patienten bei frischer Ruptur des VKB mittels DIS versorgt. Das Durchschnittsalter lag bei 30 Jahren (16 bis 64 Jahre). Nach einem Follow-up von 12 Monaten betrug die Differenz im Lachman-Test im Vergleich zur unverletzten Gegenseite 1,3 mm. Bei einem prätraumatischen Tegner-Score von 5,3 konnte nach 12 Monaten ein Score von 4,4 erreicht werden. - Abb. 8 zeigt eine Verlaufs-MRT 1 Jahr nach erfolgreicher operativer Versorgung mittels DIS. Das $\mathrm{VKB}$ ist gut abgrenzbar und anatomisch eingeheilt.

Innerhalb eines Beobachtungszeitraumes von 18 Monaten mussten 5 Patienten aufgrund einer Rezidivinstabilität revidiert werden. In 3 Fällen zeigte sich eine Reruptur, in 2 Fällen war das VKB nicht eingeheilt. In allen 5 Fällen war eine einzeitige Revision möglich. Nach Entfernung des Monoblocks konnte der tibia- 

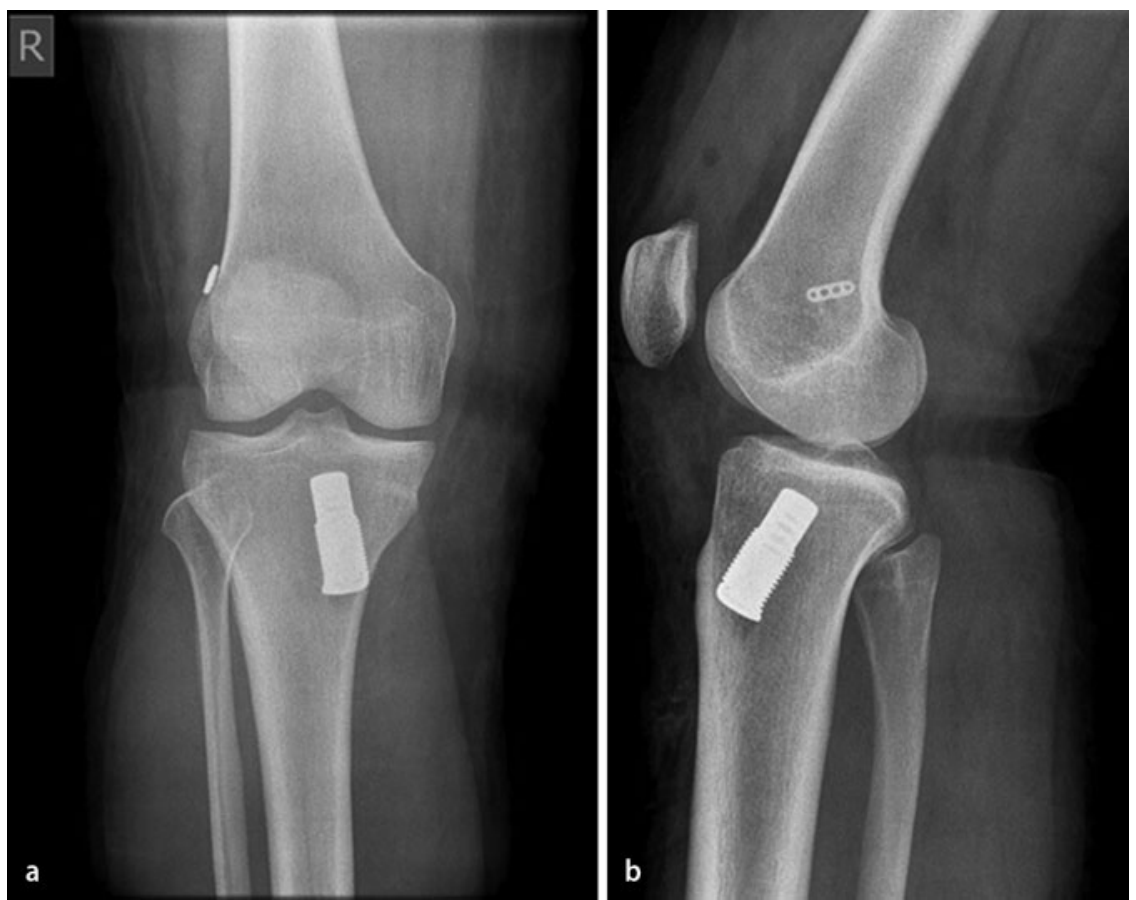

Abb. 7 A Postoperative Röntgenkontrolle im a anterioren und b seitlichen Strahlengang

le Bohrkanal anatomisch platziert werden. Die Fixation erfolgte mittels Interferenzschraube und Endobutton als Hybridfixation. Gegebenenfalls wurde der Durchmesser der Interferenzschraube größer gewählt, um eine stabile Fixation des Transplantates zu erreichen. In einem weiteren Fall erfolgte eine Revision aufgrund einer Arthrofibrose.

In unserem Patientengut zeigte sich weiterhin, dass Patienten nach DIS signifikant schneller wieder an ihren Arbeitsplatz zurückkehren als Patienten, die mittels Ersatzplastik behandelt wurden. Dies gilt auch für schwere körperliche Tätigkeit. Auch hinsichtlich der Rückkehr zur ursprünglich ausgeübten Sportart ergab sich ein ähnliches Bild: Nach DIS kehrten die Patienten signifikant schneller wieder zu ihrer Sportart zurück als nach konventioneller Ersatzplastik. Bezüglich des sportlichen Niveaus zeigten sich jedoch keine Unterschiede.

Zusammenfassend ergaben sich somit vielversprechende erste biomechanische und klinische Ergebnisse. Dennoch soll die DIS nicht als Alternative zur Ersatzplastik betrachtet werden, sondern vielmehr das operative Portfolio des in der Kreuzbandchirurgie tätigen Arztes erweitern. Ob sich auch im Langzeitverlauf Ergebnisse erreichen lassen, die denen der konventionellen Ersatzplastik vergleichbar sind, müssen weitere Studien zeigen. Ob die DIS für alle Patienten, unabhängig vom körperlichen Aktivitätsniveau, der Rupturform und weiteren Faktoren geeignet ist, unter welchen Voraussetzungen es zu einer stabilen Einheilung kommt und welche strukturellen Eigenschaften das reinserierte VKB tatsächlich hat, ist ebenfalls Gegenstand weiterer Forschung.

\section{Korrespondenzadresse}

\section{Dr. B. Schliemann}

Klinik für Unfall-, Hand- und

Wiederherstellungschirurgie

Universitätsklinikum Münster

Albert-Schweitzer Campus 1

Gebäude W1, 48149 Münster

benedikt.schliemann@ukmuenster.de

http://www.traumacentrum.de

\section{Einhaltung ethischer Richtlinien}

Interessenkonflikt. B. Schliemann, M. Herbort, S. Lenschow, M.J. Raschke und C. Kösters geben an, dass kein Interessenkonflikt besteht.

Dieser Beitrag beinhaltet keine Studien an Menschen oder Tieren.

The supplement containing this article is not sponsored by industry.

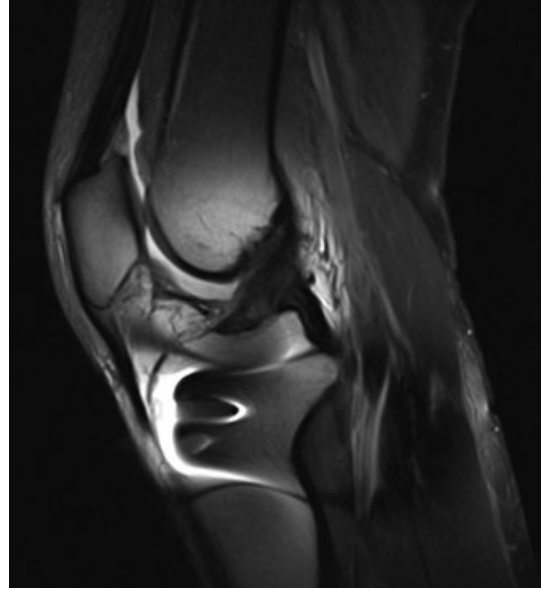

Abb. $8 \Delta$ Verlaufs-MRT 1 Jahr nach operativer Versorgung mittels dynamischer intraligamentärer Stabilisierung, die eine erfolgreiche Naht des vorderen Kreuzbandes dokumentiert

\section{Literatur}

1. Alentorn-Geli E, Lajara F, Samitier G, Cugat R (2010) The transtibial versus the anteromedial portal technique in the arthroscopic bone-patellar tendon-bone anterior cruciate ligament reconstruction. Knee Surg Sports Traumatol Arthrosc 18(8):1013-1037

2. Alentorn-Geli E, Samitier G, Alvarez P, Steinbacher G, Cugat R (2010) Anteromedial portal versus transtibial drilling techniques in ACL reconstruction: a blinded cross-sectional study at two- to five-year follow-up. Int Orthop 34(5):747-754

3. Beynnon BD, Johnson RJ, Abate JA, Fleming BC, Nichols CE (2005) Treatment of anterior cruciate ligament injuries, part 2. Am J Sports Med 33(11):1751-1767

4. Beynnon BD, Johnson RJ, Abate JA, Fleming BC, Nichols CE (2005) Treatment of anterior cruciate ligament injuries, part I. Am J Sports Med 33(10):1579-1602

5. Eggli S, Kohlhof H, Zumstein M, Henle P, Hartel M, Evangelopoulos DS, Bonel H, Kohl S (2014) Dynamic intraligamentary stabilization: novel technique for preserving the ruptured $\mathrm{ACL}$. Knee Surg Sports Traumatol Arthrosc 23(4):1215-1221

6. Heitmann M, Dratzidis A, Jagodzinski M, Wohlmuth P, Hurschler C, Puschel K, Giannakos A, Preiss A, Frosch KH (2014) [Ligament bracing-augmented cruciate ligament sutures: biomechanical studies of a new treatment concept]. Unfallchirurg 117(7):650-657

7. Heitmann M, Gerau M, Hotzel J, Giannakos A, Frosch KH, Preiss A (2014) [Ligament bracing-augmented primary suture repair in multiligamentous knee injuries]. Oper Orthop Traumatol 26(1):19-29

8. Henle P, Roder C, Perler G, Heitkemper S, Eggli S (2015) Dynamic Intraligamentary Stabilization (DIS) for treatment of acute anterior cruciate ligament ruptures: case series experience of the first three years. BMC Musculoskelet Disord 16:27. doi:10.1186/s12891-015-0484-7

9. Johnson RJ, Beynnon BD, Nichols CE, Renstrom PA (1992) The treatment of injuries of the anterior cruciate ligament. J Bone Joint Surg Am 74(1):140151 
10. Kohl S, Evangelopoulos DS, Kohlhof $H$, Hartel $M$, Bonel H, Henle P, von Rechenberg B, Eggli S (2013) Anterior crucial ligament rupture: self-healing through dynamic intraligamentary stabilization technique. Knee Surg Sports Traumatol Arthrosc 21(3):599-605

11. Kösters C, Herbort M, Schliemann B et al (2015) Dynamische intraligamentäre Stabilisierung des vorderen Kreuzbandes. Operationstechnik und klinische Kurzzeitergebnisse. Unfallchirurg 118:364371

12. Lubowitz JH (2014) Anatomic ACL reconstruction produces greater graft length change during knee range-of-motion than transtibial technique. Knee Surg Sports Traumatol Arthrosc 22(5):1190-1195

13. Marshall JL, Warren RF, Wickiewicz TL (1982) Primary surgical treatment of anterior cruciate ligament lesions. Am J Sports Med 10(2):103-107

14. Marshall JL, Warren RF, Wickiewicz TL, Reider B (1979) The anterior cruciate ligament: a technique of repair and reconstruction. Clin Orthop Relat Res 143:97-106

15. Paessler HH, Deneke J, Dahners LE (1992) Augmented repair and early mobilization of acute anterior cruciate ligament injuries. Am J Sports Med 20(6):667-674 